ARTICLE

\title{
Lead iodide perovskite light-emitting field-effect transistor
}

\author{
Xin Yu Chin ${ }^{1}$, Daniele Cortecchia 2,3 , Jun Yin ${ }^{1,4}$, Annalisa Bruno ${ }^{1,3} \&$ Cesare Soci, ${ }^{1,4}$
}

Despite the widespread use of solution-processable hybrid organic-inorganic perovskites in photovoltaic and light-emitting applications, determination of their intrinsic charge transport parameters has been elusive due to the variability of film preparation and history-dependent device performance. Here we show that screening effects associated to ionic transport can be effectively eliminated by lowering the operating temperature of methylammonium lead iodide perovskite $\left(\mathrm{CH}_{3} \mathrm{NH}_{3} \mathrm{Pbl}_{3}\right)$ field-effect transistors. Field-effect carrier mobility is found to increase by almost two orders of magnitude below $200 \mathrm{~K}$, consistent with phonon scattering-limited transport. Under balanced ambipolar carrier injection, gate-dependent electroluminescence is also observed from the transistor channel, with spectra revealing the tetragonal to orthorhombic phase transition. This demonstration of $\mathrm{CH}_{3} \mathrm{NH}_{3} \mathrm{Pbl}_{3}$ light-emitting field-effect transistors provides intrinsic transport parameters to guide materials and solar cell optimization, and will drive the development of new electro-optic device concepts, such as gated light-emitting diodes and lasers operating at room temperature.

\footnotetext{
${ }^{1}$ Division of Physics and Applied Physics, School of Physical and Mathematical Sciences, Nanyang Technological University, 21 Nanyang Link, Singapore 637371, Singapore. ${ }^{2}$ Interdisciplinary Graduate School, Nanyang Technological University, Singapore 639798, Singapore. ${ }^{3}$ Energy Research Institute @ NTU (ERI@N), Research Techno Plaza, Nanyang Technological University, 50 Nanyang Drive, Singapore 637553, Singapore. ${ }^{4}$ Centre for Disruptive Photonic Technologies, Nanyang Technological University, Nanyang, 21 Nanyang Link, Singapore 637371, Singapore. Correspondence and requests for materials should be addressed to C.S. (email: csoci@ntu.edu.sg).
} 
rganolead halide perovskites are emerging solutionprocessable materials with outstanding optoelectronic properties $^{1-7}$. Among them, methylammonium lead iodide $\mathrm{CH}_{3} \mathrm{NH}_{3} \mathrm{PbI}_{3}$ has proven to be an exceptional light harvester for hybrid organic-inorganic solar cells ${ }^{3,8-15}$, which in just 4 years achieved an impressive National Renewable Energy Laboratory (NREL)-certified power conversion efficiency of $20.1 \%$, and remarkable performance in a variety of device architectures ${ }^{16}$. Thanks to their cost-effectiveness and ease of processing, hybrid perovskites have naturally attracted a vast interest for applications beyond photovoltaic energy conversion, such as water splitting ${ }^{17}$, light-emitting diodes ${ }^{18-20}$ and tunable, electrically pumped lasers ${ }^{6,21-23}$. So far, transport parameters of perovskite materials were mostly deduced from the study of photovoltaic devices, which indicated ambipolar transport ${ }^{3,24,25}$, of holes and electrons within the perovskite active region, and long electron-hole pair diffusion length ${ }^{4,5,26}$

First-principle calculations for this class of materials predict that hole mobility is up to $3,100 \mathrm{~cm}^{2} \mathrm{~V}^{-1} \mathrm{~s}-1$ and electron mobility is $1,500 \mathrm{~cm}^{2} \mathrm{~V}^{-1} \mathrm{~s}^{-1}$ with concentration of $10^{16} \mathrm{~cm}^{-3}$ at $400 \mathrm{~K}$ (ref. 27), and high-frequency mobility of $8 \mathrm{~cm}^{2} \mathrm{~V}^{-1} \mathrm{~s}^{-1}$ was determined in $\mathrm{CH}_{3} \mathrm{NH}_{3} \mathrm{PbI}_{3}$ spin-coated thin film by $\mathrm{THz}$ spectroscopy $^{28}$, a remarkably high value for solution-processed materials. A combination of resistivity and Hall measurement further revealed that the mobility of $\sim 66 \mathrm{~cm}^{2} \mathrm{~V}^{-1} \mathrm{~s}-1$ is achievable in $\mathrm{CH}_{3} \mathrm{NH}_{3} \mathrm{PbI}_{3}$ (ref. 29).

Recent publications on organolead halide perovskite single crystals also reported extremely low trap densities, leading to a noticeable enhancement of photogenerated carrier lifetime and mobility ${ }^{30-32}$.

However, very recently ion drift was shown to play a dominant role on charge transport properties ${ }^{33}$, stimulating an ongoing debate about the carrier character and the origin of anomalous hysteresis, together with the role of polarization, ferroelectric and trap-state filling effects in organolead halide perovskite devices investigated at room temperature ${ }^{34-37}$.
Despite the rapid advancement of optoelectronic applications, a big gap remains in understanding the fundamental transport properties of organolead halide perovskites, namely charge carrier character, mobility and charge transport mechanisms. To fill this gap, studies of basic field-effect transistor (FET) devices are urgently needed. Historically, related tin(II)-based 2D hybrid perovskites have attracted major interest for FET fabrication because of their attractive layered structure, with demonstrated field-effect mobilities up to $0.62 \mathrm{~cm}^{2} \mathrm{~V}^{-1} \mathrm{~s}^{-1}$ and $I_{\mathrm{on}} / I_{\text {off }}$ ratio above $10^{4}$ (ref. 38). Improvement of mobility can be achieved by substitution of organic cation in hybrid perovskite, yielding FET saturation-regime mobility as high as $1.4 \mathrm{~cm}^{2} \mathrm{~V}^{-1} \mathrm{~s}^{-1}$, and nearly an order of magnitude lower linear-regime mobility ${ }^{39}$. Further improvement was demonstrated through melt processed deposition technique, where saturation and linear mobilities of 2.6 and $1.7 \mathrm{~cm}^{2} \mathrm{~V}^{-1} \mathrm{~s}^{-1}$ with $I_{\text {on }} / I_{\text {off }}$ of $10^{6}$ were achieved ${ }^{40}$. Conversely, only rare examples of $3 \mathrm{D}$ hybrid perovskites FETs can be found in the literature (see Supplementary Information in ref. 15), with limited hole mobility of the order of $\sim 10^{-5} \mathrm{~cm}^{2} \mathrm{~V}^{-1} \mathrm{~s}^{-1}$ in the case of $\mathrm{CH}_{3} \mathrm{NH}_{3} \mathrm{PbI}_{3}$ and strong hysteresis due to ionic transport, which so far have hindered the development of FET applications. Nonetheless, high photoluminescence efficiency ${ }^{22}$ and widely tunable band gap from visible to infrared ${ }^{29,41}$ make $\mathrm{CH}_{3} \mathrm{NH}_{3} \mathrm{PbI}_{3}$ extremely attractive for the fabrication of solution processable lightemitting field-effect transistors (LE-FET), a device concept that may be integrated in heterogeneous optolectronic systems, such as flexible electroluminescent displays ${ }^{42}$ or electrically pumped lasers $^{43}$.

Here we report the fabrication and characterization of $\mathrm{CH}_{3} \mathrm{NH}_{3} \mathrm{PbI}_{3}$ FETs, and their operation as light-emitting FETs yielding gate-assisted electroluminescence (EL). Lowtemperature measurements were used to effectively remove screening effects arising from ionic transport, allowing the determination of intrinsic transport parameters such as carrier density and mobility. Field-effect mobility of $\mathrm{CH}_{3} \mathrm{NH}_{3} \mathrm{PbI}_{3}$ is a

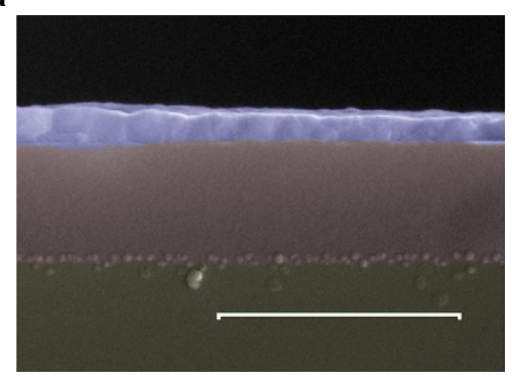

C

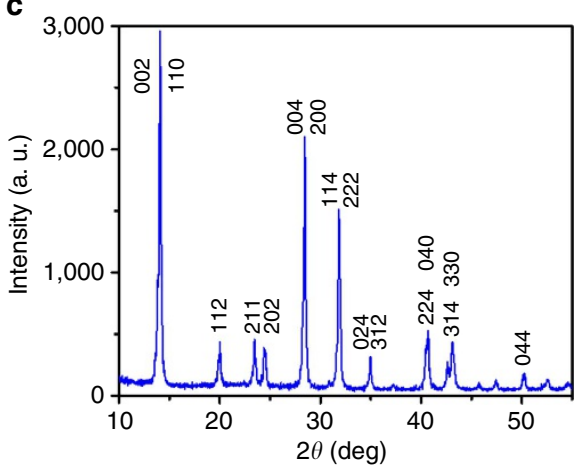

b

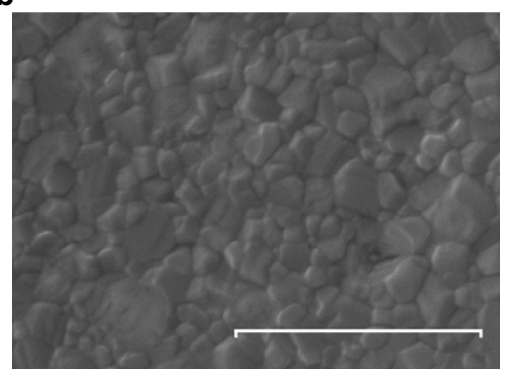

d

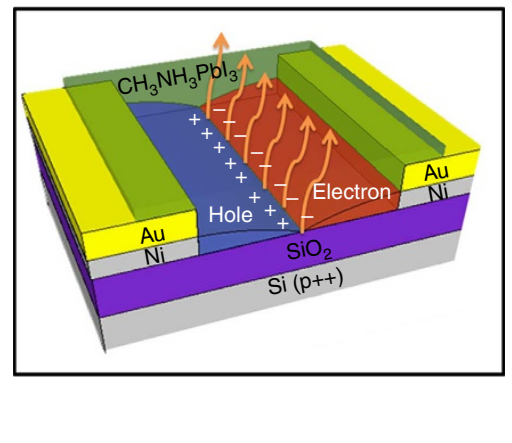

Figure 1 | FET device configuration and thin-film characterization. $(\mathbf{a}, \mathbf{b})$ Cross-sectional (a) and top-view (b) scanning electron miscroscope micrographs of the $\mathrm{CH}_{3} \mathrm{NH}_{3} \mathrm{Pbl}_{3}$ thin film. Scale bars, $1 \mu \mathrm{m}$. (c) X-ray diffraction pattern of $\mathrm{CH}_{3} \mathrm{NH}_{3} \mathrm{Pbl}_{3}$ film on $\mathrm{SiO}_{2} / \mathrm{Si}(\mathrm{p}++$ ) substrate, confirming the tetragonal structure of the perovskite and space group $14 / \mathrm{mcm}$. (d) Schematic of the bottom-gate, bottom contact LE-FET configuration used in this study. 
found to increase by almost two orders of magnitude from room temperature down to $78 \mathrm{~K}$, a behaviour consistent with phonon scattering-limited transport of conventional inorganic semiconductors. We also confirm the ambipolar nature of charge transport in $\mathrm{CH}_{3} \mathrm{NH}_{3} \mathrm{PbI}_{3}$, which yields close to ideal ambipolar transistor characteristics and EL from the transistor channel under balanced injection conditions. This demonstration of $\mathrm{CH}_{3} \mathrm{NH}_{3} \mathrm{PbI}_{3}$ light-emitting FETs provides an essential guideline for materials optimization through chemical synthesis and future improvements of solar cell performance. And the novel device concept opens up new opportunities for the development of electro-optic devices based on $\mathrm{CH}_{3} \mathrm{NH}_{3} \mathrm{PbI}_{3}$, such as gated lightemitting devices.

\section{Results}

Thin film characterization. Deposition methods of solutionprocessed organo-lead hybrid perovskite have direct consequences on the morphology of thin film, hence on charge transport properties of the material ${ }^{2}$. Here we used the solvent engineering technique recently reported for optimized solar cell fabrication ${ }^{14}$ to deposit a compact and uniform $\mathrm{CH}_{3} \mathrm{NH}_{3} \mathrm{PbI}_{3}$ perovskite layer $(\sim 150 \mathrm{~nm}$ thick) on top of heavily p-doped $\mathrm{Si}$ with thermally grown $\mathrm{SiO}_{2}$ (Fig. 1a). The resulting thin films are of very high quality: they consist of closely packed, large domains with grain size up to $200 \mathrm{~nm}$, as seen in the top view scanning electron microscope (SEM) image in Fig. 1b. They crystallize in a perfect tetragonal structure, as revealed by the X-ray diffraction analysis in Fig. 1c. A film roughness of $R_{\mathrm{RMS}}=10.8 \mathrm{~nm}$ was evaluated by atomic force microscopy (AFM; Supplementary Fig. 1). Availability of such high-quality films is essential to minimize the influence of metal contacts and charge carrier scattering across the film, so as to obtain intrinsic transport parameters from FET measurements. The device structure used in this study is shown in Fig. 1d. A bottom gate, bottom contact configuration was employed to allow deposition of active materials to be the last step in the fabrication. This is to minimize exposure of $\mathrm{CH}_{3} \mathrm{NH}_{3} \mathrm{PbI}_{3}$ to moisture in the environment, and to avoid potential overheating during the metal electrode deposition.

Low-temperature FET characterization. As reported in the literature, transport characteristics of flat-junction $\mathrm{CH}_{3} \mathrm{NH}_{3} \mathrm{PbI}_{3}$ solar cells are often subject to strong hysteresis ${ }^{44}$, which so far hindered a complete understanding of the electrical response, and the determination of intrinsic transport parameters of the perovskite $^{34-36}$. The origin of this anomalous behaviour has been attributed to capacitive effects associated with ferroelectricity arising from the spontaneous polarization of methylammonium cation and lattice distortion effects, diffusion of excess ions as interstitial defects, and trapping/de-trapping of charge carriers at the interface ${ }^{34-36,44}$. Photocurrent hysteresis in $\mathrm{CH}_{3} \mathrm{NH}_{3} \mathrm{PbI}_{3}$ planar heterojunction solar cells was found to originate from trap states on the surface and grain boundaries of the perovskite materials, which can be effectively eliminated by fullerene passivation ${ }^{36}$. Recently, hysteresis-free photovoltaic devices with well-reproducible PCEs were achieved in single crystals ${ }^{30,31}$ and millimeter-scale grain size thin films ${ }^{32}$. Piezoelectric microscopy revealed the reversible switching of the ferroelectric domains by poling with DC biases ${ }^{45}$, but a recent observation of field-switchable photovoltaic effect suggested that ion drift
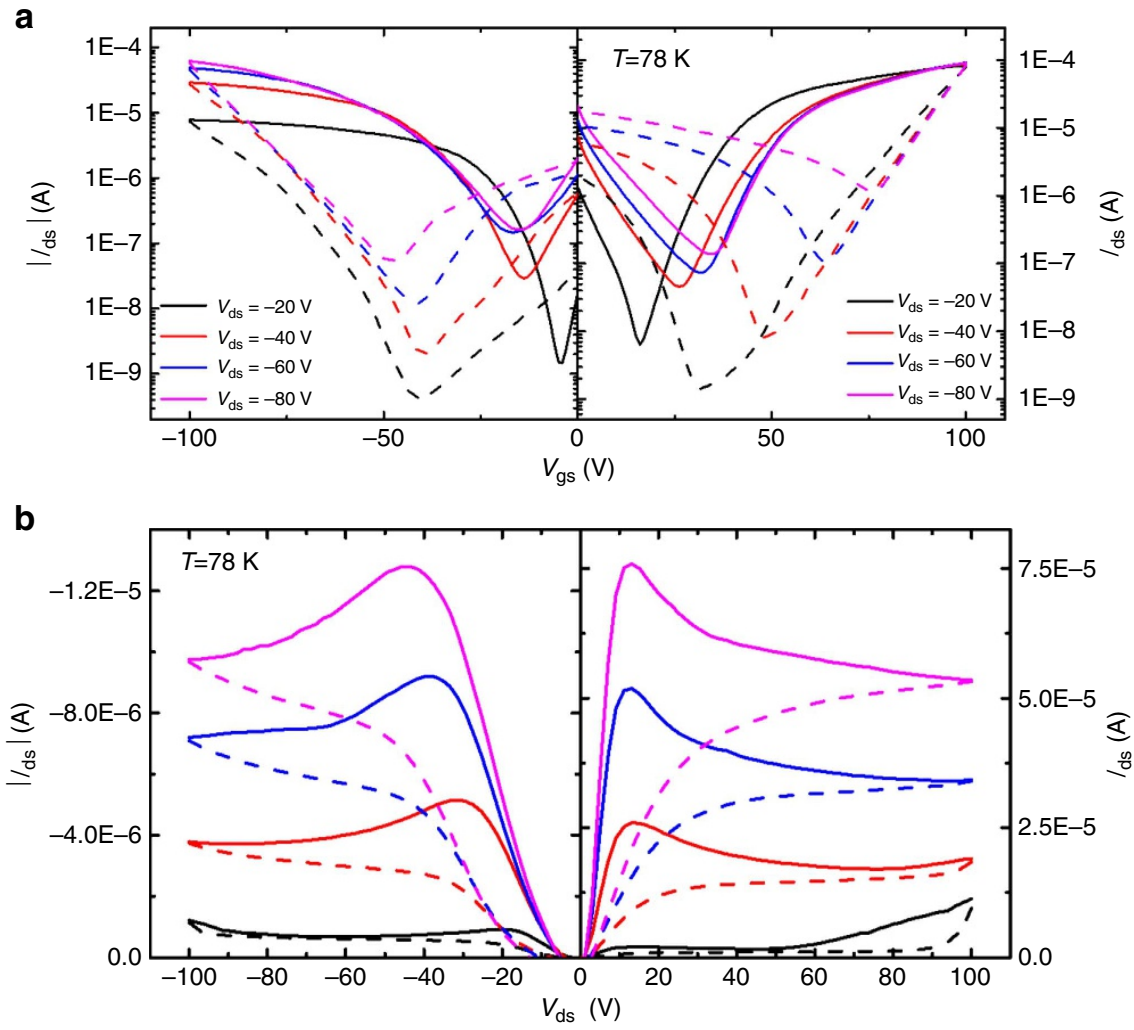

Figure 2 | FET characteristics. (a,b) Transfer (a) and output (b) characteristics obtained at $78 \mathrm{~K}$. The n-type output characteristics (right panel) were measured at $V_{\mathrm{gs}}=40-100 \mathrm{~V}\left(V_{\mathrm{gs}}=40 \mathrm{~V}\right.$ black, $V_{\mathrm{gs}}=60 \mathrm{~V}$ red, $V_{\mathrm{gs}}=80 \mathrm{~V}$ blue, $V_{\mathrm{gs}}=100 \mathrm{~V}$ magenta $)$, whereas the p-type output characteristics (left panel) are measured at $V_{g s}=-40 \mathrm{~V}$ to $-100 \mathrm{~V}\left(V_{g s}=-40 \mathrm{~V}\right.$ black, $V_{g s}=-60 \mathrm{~V}$ red, $V_{g s}=-80 \mathrm{~V}$ blue, $V_{g s}=-100 \mathrm{~V}$ magenta). Solid and dashed curves are measured with forward and backward sweeping, respectively. See Supplementary Information for the full set of FET characteristics as a function of temperature. 
under the electric field in the perovskite layer induces the formation of $\mathrm{p}-\mathrm{i}-\mathrm{n}$ structures ${ }^{33}$, as observed by electron beaminduced current measurement and Kelvin probe force microscopy ${ }^{24,25}$. A weakened switchable photovoltaic effect at low temperature and the lack of photovoltage dependence with respect to the lateral electrode spacing suggest that ferroelectric photovoltaic effect may not play dominant role in the observed field-switchable photovoltaic behaviour ${ }^{33}$. Theoretical calculations further reveal that charged $\mathrm{Pb}, \mathrm{I}$ and methylammonium vacancies have low formation energies ${ }^{40,41}$, suggesting that the high ionicity of this materials may lead to pand $\mathrm{n}$-type self-doping.

We found that reducing the operating temperature of our devices is an effective way to reduce hysteresis effects due to ionic transport/screening, allowing to record transport characteristics typical of conventional ambipolar semiconductor FETs (Fig. 2). The complete temperature evolution of ambipolar FET characteristics, from room temperature down to $78 \mathrm{~K}$, is provided in Supplementary Figs 2 and 3 of the Supplementary Information. While above $198 \mathrm{~K}$ the output characteristics show either weak or no gate voltage dependence, at and below $198 \mathrm{~K}$ the devices display 'textbook' n-type output characteristics. Similarly, typical p-type output characteristics are observed at $98 \mathrm{~K}$ and lower temperatures (Fig. 2b and Supplementary Fig. 3). Both p- and n-type transfer characteristics are independent of gate field from room temperature down to $258 \mathrm{~K}$. This is reflected in the measurement by large hysteresis loops, which do not close when transitioning from the hole- to the electron-dominated transport gate voltage ranges and vice versa. Below $258 \mathrm{~K}$, however, both $\mathrm{n}$ - and p-type transfer characteristics show a closed hysteresis loop. Hysteresis of $\mathrm{n}$ - and $\mathrm{p}$-type transfer characteristics is substantially reduced below 198 and $98 \mathrm{~K}$, respectively, consistent with the observation of ambipolar output characteristics (Fig. 2b and Supplementary Fig. 3). Induced carrier density of $\sim 3.8 \times 10^{16} \mathrm{~cm}^{-2}$, maximum $I_{\mathrm{on}} / I_{\mathrm{off}} \sim 10^{5}$ and current density of $\sim 830 \mathrm{~A} \mathrm{~cm}^{-2}$ (estimated for a $\sim 2 \mathrm{~nm}$ accumulation layer thickness) are obtained from standard transistor analysis at $198 \mathrm{~K}$. These values are comparable to those previously reported for $2 \mathrm{D}$ hybrid organic-inorganic perovskites characterized at room temperature ${ }^{39,40}$. Note that, although our low-temperature measurements clearly demonstrate the ambipolar nature of $\mathrm{CH}_{3} \mathrm{NH}_{3} \mathrm{PbI}_{3}$, previous studies have shown that carrier concentration can vary by up to six orders of magnitudes depending on the ratio of the methylammonium halide and lead iodine precursors and thermal annealing conditions, thus resulting in preferential p-type or n-type transport characteristics ${ }^{46}$.

Temperature-dependent electron and hole mobilities were extracted from the forward sweeping of transfer characteristics at $V_{\mathrm{ds}}=20 \mathrm{~V}$ and $V_{\mathrm{ds}}=-20 \mathrm{~V}$ using the standard transistor equation at linear regime ${ }^{47}$. The resulting values are shown in Fig. 3a. Note that mobilities were not extracted from backward sweeping curves to avoid misleading results due to the large hysteresis. Also, mobilities at higher $V_{\mathrm{ds}}$ (that is, in the saturation regime) were not extracted due to the difficulty to identify linear and saturation regimes at all investigated temperatures. A statistical analysis of the distribution of mobility values extracted from independent measurements across four different devices is shown in Supplementary Fig 4. Although some variability in the absolute values of electron and hole mobilities is observed from device to device, their relative magnitude and temperature dependence show consistent trends. From Fig. 3a, both electron and hole mobilities increase by a factor of $\sim 100$ from room temperature to $198 \mathrm{~K}$. Below $198 \mathrm{~K}$, there is no further improvement of electron mobility, whereas hole mobility shows an additional tenfold increase. We attribute the improvement of mobility at low temperature to the removal of screening effects arising from the ionic transport of methylammonium cations. The phonon energy of methylammonium cation was estimated to be $\sim 14.7 \mathrm{meV}$ from previous combination of density function theory (DFT) and Raman studies ${ }^{48}$. The observation of weak improvement of field-effect mobilities below $198 \mathrm{~K}$ $\left(E_{\text {thermal }}=16.7 \mathrm{meV}\right)$ is therefore consistent with the quenching of phonon interactions related to the organic cations. This is also in agreement with the weakening of field-switchable photovoltaic effects at low temperature ${ }^{33}$, strongly suggesting that field-effect transport is phonon limited at room temperature. Despite the remarkable improvement of field-effect mobilities, hysteresis was not completely removed at the lowest temperature investigated. This could be due to the untreated semiconductor-dielectric interface, which is known to affect semiconductor film morphology, number of trap states and surface dipoles, similar to the case of organic FET devices ${ }^{47}$. The reduction of trap density in single crystal $^{30,31}$ and large grain size thin films ${ }^{32}$ enormously enhances stability of photovoltaic devices. Thus, improvement of bulk crystallinity is also expected to reduce hysteresis of FETs, with proper control of the morphology of the semiconductor-dielectric interface, where the nanometre thin field-effect transport channel is created ${ }^{47}$. Both hole and electron mobilities extracted in the linear regime at $78 \mathrm{~K}$ are slightly smaller than the corresponding saturation regime mobilities $\left(\mu_{\mathrm{e} \text {,inear }} / \mu_{\mathrm{e} \text {,saturation }}=6.7 \times 10^{-2} / 7.2 \times 10^{-2} \mathrm{~cm}^{2} \mathrm{~V}^{-1} \mathrm{~s}^{-1}\right.$ and $\mu_{\mathrm{h}, \text { linear }} / \mu_{\mathrm{h} \text {,saturation }}=6.6 \times 10^{-3} / 2.1 \times 10^{-2} \mathrm{~cm}^{2} \mathrm{~V}^{-1} \mathrm{~s}^{-1}, \quad$ extracted at $V_{\mathrm{ds}}= \pm 20 \mathrm{~V}$ for linear regime and $V_{\mathrm{ds}}= \pm 80 \mathrm{~V}$ for saturation regime from Fig. 2a). A previous study of spin-coated hybrid perovskite channels indicated linear regime mobility values 1 to 2 orders of magnitude lower than in the saturation regime ${ }^{39}$. The suppression of the linear regime mobility is presumably associated to grain-boundary effects, which give rise to a large concentration of traps. Thus, our reported linear regime mobilities set a lower limit for electron and hole mobilities of $\mathrm{CH}_{3} \mathrm{NH}_{3} \mathrm{PbI}_{3}$.

DFT modelling and mobility computation. To better understand the transport data, we estimated the mobility of $\mathrm{CH}_{3} \mathrm{NH}_{3} \mathrm{PbI}_{3}$ for both tetragonal and orthorhombic crystallographic phases using semi-classical Boltzmann transport theory ${ }^{49}$, upon deducing charge carrier effective masses and electron (hole)-phonon coupling. Electron and hole effective masses listed in Supplementary Table 1 were derived by quadratic fitting of the band structure dispersion (Fig. 3c,d); the corresponding fitting parameters are summarized in Supplementary Table 2. The average effective mass of electrons (tetragonal: $0.197 m_{0}$, orthorhombic: $0.239 m_{0}$ ) is consistently smaller than the one of holes (tetragonal: $0.340 m_{0}$, orthorhombic: $0.357 m_{0}$ ), similar to a previous report ${ }^{48,50}$. The resulting mobilities (Fig. 3b) increase at lower temperatures due to the Boltzmann activation energy (see Computational Methods section), in agreement with the trend of our experimental results. Although the calculated mobilities are substantially larger than the experimental values in Fig. 3a, calculations reflect fairly well the relative magnitude of electron versus hole mobility, as well as the different mobility of the two crystallographic phases. Within the entire temperature range investigated, electron mobilities exceed hole mobilities by approximately a factor of two, and increase by nearly one order of magnitude below the phase transition temperature $\left(\mu_{\mathrm{e}}=2,577-11,249 \mathrm{~cm}^{-2} \mathrm{~V}^{-1} \mathrm{~s}^{-1}\right.$ and $\mu_{\mathrm{h}}=1,060-4,630 \mathrm{~cm}^{-2} \mathrm{~V}^{-1} \mathrm{~s}^{-1}$ for the orthorhombic phase and $\mu_{\mathrm{e}}=466-2,046 \mathrm{~cm}^{-2} \mathrm{~V}^{-1} \mathrm{~s}^{-1}$ and $\mu_{\mathrm{h}}=140$ $-614 \mathrm{~cm}^{-2} \mathrm{~V}^{-1} \mathrm{~s}^{-1}$ for the tetragonal phase). The small experimental values can be partly attributed to the increase of effective masses by elastic carrier - phonon scattering, which is 

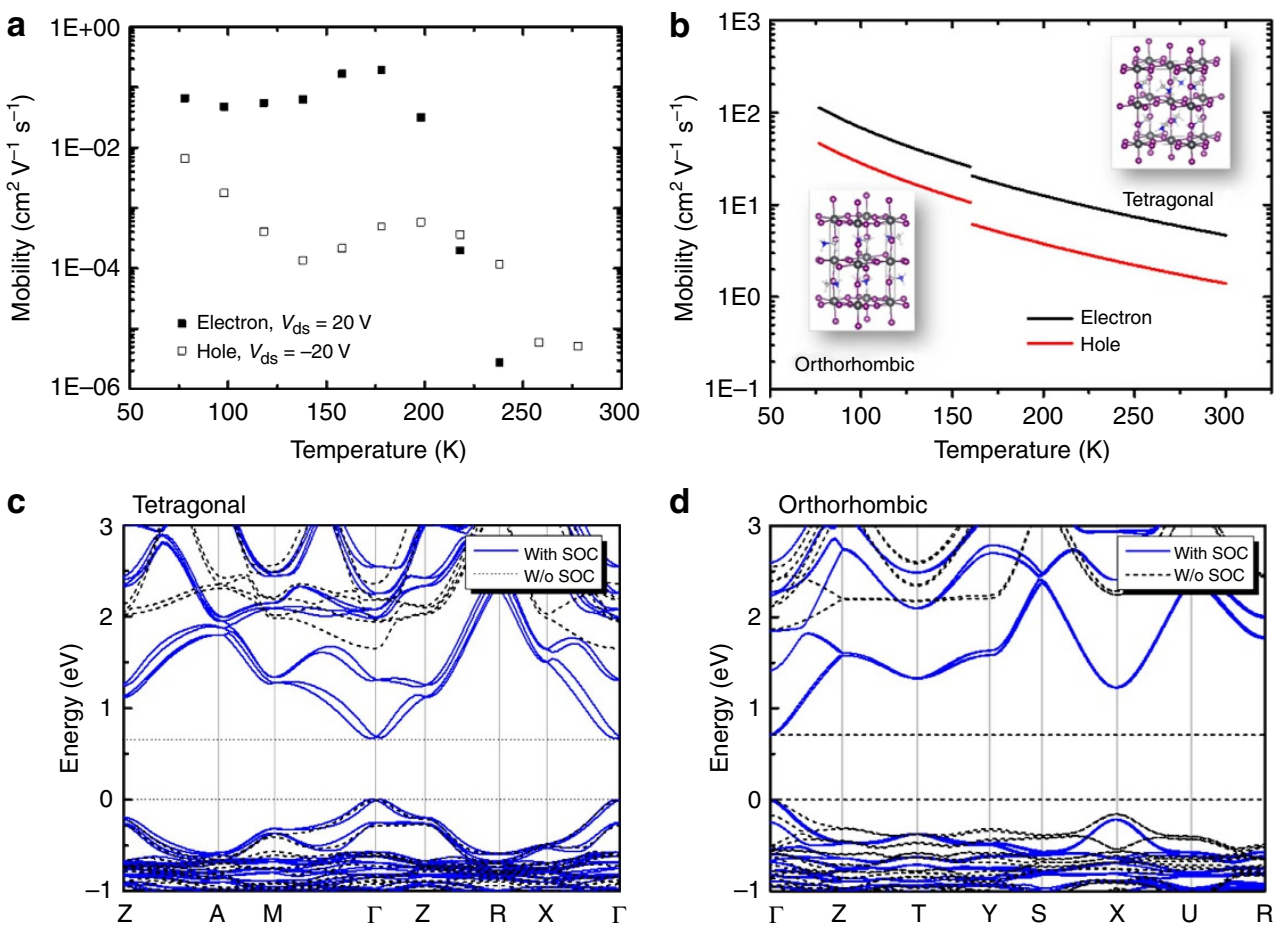

Figure 3 | Experimental and theoretical field-effect mobility and band structures of $\mathbf{C H}_{\mathbf{3}} \mathbf{N H}_{\mathbf{3}} \mathbf{P b} \mathbf{3}_{\mathbf{3}}$. (a) Temperature dependence of field-effect electron and hole mobilities, extracted from the forward sweeping of transfer characteristics at $V_{\mathrm{ds}}=20 \mathrm{~V}$ and $V_{\mathrm{ds}}=-20 \mathrm{~V}$, respectively. (b) Calculated temperature dependence hole (red curves) and electron (black curves) mobility in tetragonal ( $T=300$ to $160 \mathrm{~K}$ ) and orthorhombic ( $T=160$ to $77 \mathrm{~K}$ ) phases of $\mathrm{CH}_{3} \mathrm{NH}_{3} \mathrm{Pbl}_{3}$. The crystal unit cells of the two phases are shown as insets. (c, d) Band structures of the tetragonal (c) and orthorhombic (d) phases obtained by DFT-Perdew-Burke-Ernzerhof method with (solid curves) and without (dotted curves; W/o) spin-orbital coupling (SOC).

expected in real crystals because of defects and disorder induced by the organic components, as well as carrier-carrier scattering at high-electron and hole concentrations ${ }^{51}$. Formation of segregation pathways for hole and electron transport owing to the ferroelectric methylammonium cation could also elongate the carrier drifting path, hence lower carrier mobilities ${ }^{52}$. In addition, polycrystalline domains typical of solution-processed $\mathrm{CH}_{3} \mathrm{NH}_{3} \mathrm{PbI}_{3}$ thin films (Fig. 1a,b) are likely to weaken the electronic coupling between grains, requiring charge carriers to hop along and across domain boundaries, further reducing the effective carrier mobility. This is consistent with observation of giant photoinduced modulation of the dielectric constant, which was attributed to localized polaron hopping with relatively small activation energy ${ }^{53}$.

EL characteristics of LE-FET. The excellent ambipolar characteristics shown by the $\mathrm{CH}_{3} \mathrm{NH}_{3} \mathrm{PbI}_{3}$ FET at low temperature (Fig. 2) are rather encouraging for the realization of light-emitting devices operating under balanced carrier injection ${ }^{6,18-23}$. In particular, large carrier injection via charge accumulation at the semiconductor-dielectric interface is known to be an effective way to achieve bright and fast-switchable EL, and to optimize the spatial location of the carrier recombination zone in organic gateassisted LE-FETs ${ }^{54,55}$. In LE-FET devices, ambipolar channels are formed simultaneously by proper source-drain and gate biasing. Under perfectly balanced conditions, holes and electrons injected from opposite electrodes recombine in the middle of the FET channel, thus defining a very narrow radiative emission zone, as depicted in Fig. 1d. The brightness of emission as well as the spatial position of the radiative recombination zone can be tuned by gate and drain-source biases ${ }^{47}$. LE-FET structures have proved to improve the lifetime and efficiency of light-emitting materials, thanks to the large electrical injection achievable, and the possibility to optimize and balance charge carrier recombination compared with conventional LED devices ${ }^{42,56}$. Combined with the ease of integration as nanoscale light sources in optoelectronic and photonic devices, this makes LE-FETs a very promising concept for applications in optical communication systems, solid-state lighting and electrically pumped lasers $^{56,57}$.

Indeed, our $\mathrm{CH}_{3} \mathrm{NH}_{3} \mathrm{PbI}_{3}$ FETs emit light when operated in their ambipolar regime at low temperature (78-178 K). Typical EL spectra are displayed in Fig. 4. Note that no light emission could be observed above $198 \mathrm{~K}$, most likely due to the ionic screening effects discussed earlier, so that low-temperature operation is necessary at this stage. Ionic screening is likely to be reduced in films with higher crystallinity as those recently reported $^{30,31}$, potentially enabling perovskite LE-FET operation up to room temperature. The emission spectra of the LE-FET are consistent with direct recombination of injected electrons and holes into the perovskite-active region. At the lowest temperature investigated $(78 \mathrm{~K})$, the EL spectrum shows three peaks centred at $750 \mathrm{~nm}$ (Peak 1), $780 \mathrm{~nm}$ (Peak 2) and $800 \mathrm{~nm}$ (Peak 3), with distinct amplitudes and spectral positions at the various temperatures. Although Peak 1 and Peak 3 appear only below $158 \mathrm{~K}$, Peak 2 dominates the EL spectra at higher temperatures. A similar behaviour was very recently observed in photoluminescence spectra of $\mathrm{CH}_{3} \mathrm{NH}_{3} \mathrm{PbI}_{3}$ films and single crystals ${ }^{58,59}$, and related to the structural transition from a lowtemperature orthorhombic phase to a high-temperature tetragonal phase occurring around $162 \mathrm{~K}$. Occurrence of this phase transition is predicted by density functional theory ${ }^{60,61}$ (see also DFT calculations in Fig. 3c,d) and was confirmed to occur in the temperature ranges of $150-170 \mathrm{~K}$ for $\mathrm{CH}_{3} \mathrm{NH}_{3} \mathrm{PbI}_{3}$ and $120-140 \mathrm{~K}$ for hybrid $\mathrm{CH}_{3} \mathrm{NH}_{3} \mathrm{PbI}_{3-\mathrm{x}} \mathrm{Cl}_{\mathrm{x}}$ by light absorption studies ${ }^{6}$. Thus, their characteristic temperature dependence suggests that Peak 1 and Peak 3 in our EL measurements are due to bound excitons in the low-temperature orthorhombic 


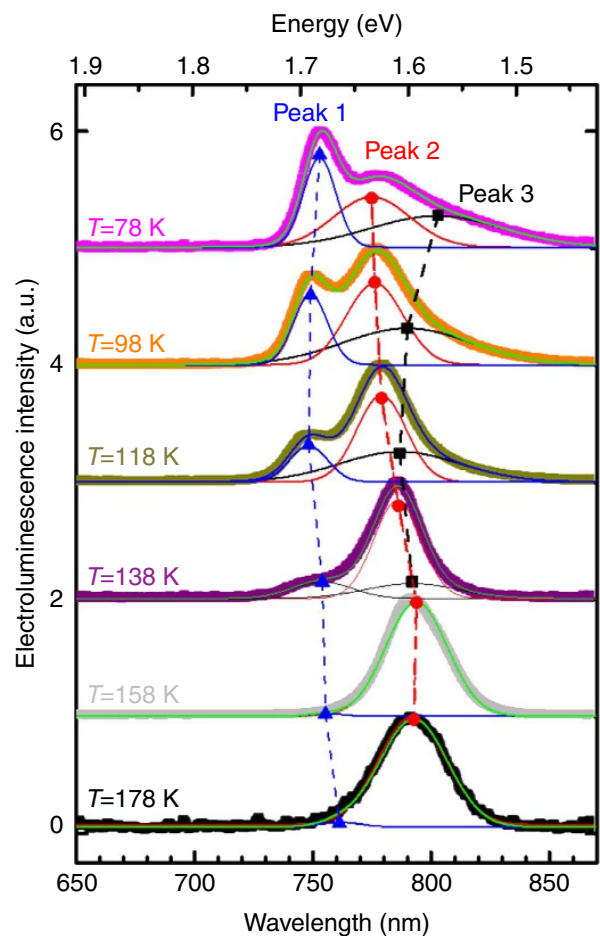

Figure 4 | Low-temperature electroluminescence spectra of $\mathrm{CH}_{3} \mathrm{NH}_{3} \mathrm{Pbl}_{3}$ LE-FET. EL spectra collected at $V_{\mathrm{ds}}=100 \mathrm{~V}, V_{\mathrm{gs}}=100 \mathrm{~V}$, normalized to their maximum peak. The spectra were fitted by three Gaussian curves (solid lines). The shift in peak position of the $750 \mathrm{~nm}$ peak (Peak 1, blue triangles), the $780 \mathrm{~nm}$ peak (Peak 2, red circles) and the $800 \mathrm{~nm}$ peak (Peak 3, black squares) is indicated by the dashed lines.

phase, whereas Peak 2 may be related to free excitons in the high temperature, smaller bandgap tetragonal phase ${ }^{59}$. To quantify the relative intensity and spectral energy of the three emission peaks as a function of temperature, we analysed the EL spectra by a deconvoluted Gaussian fitting (see Gaussian curves in Fig. 4 and corresponding fitted parameters in Supplementary Fig. 5). Although Peak 1 shows the expected blue shift at the lowest temperatures, its temperature dependence in the intermediate range $118-178 \mathrm{~K}$ is rather complicated (Supplementary Fig. 5a). Peak 2 position slightly blue shifts over the whole temperature region, whereas Peak 3 shows a significant red shift in the 138-78 K region. Moreover, although the Gaussian full-width at half-maximum of Peak 1 reduces at lower temperatures, the full-width at half-maximum of Peak 2 and Peak 3 shows the opposite behaviour (Supplementary Fig. 5b), as previously seen in low-temperature photoluminescence measurements ${ }^{58,59}$. At this stage, the anomalous spectral shift and broadening of the EL peaks and the nature of the three peaks as a function of temperature are not completely understood, and further investigations are needed to reveal their nature.

Light emission from FET channel. To achieve simultaneous hole and electron injection in a LE-FET, the local gate potential at drain and source electrodes must be larger than the threshold voltage of either of the charge carrier (that is, $\left|V_{\mathrm{d}}\right|>\left|V_{\mathrm{th}, \mathrm{h}}\right|$ and $V_{\mathrm{s}}>V_{\text {th,e }}$, or $V_{\mathrm{d}}>V_{\text {th,e }}$ and $\left.\left|V_{\mathrm{s}}\right|>\left|V_{\mathrm{th}, \mathrm{h}}\right|\right)^{47}$. Under this condition, drain-source and gate voltages are tuned to control the injected current density of both carriers, which manipulate the spatial position of the emission zone as well as the EL intensity ${ }^{47}$. Figure 5 shows microscope images of the emission zone of the LE-FET recorded at $158 \mathrm{~K}$ under different biasing conditions. Despite the grainy light emission pattern due to the

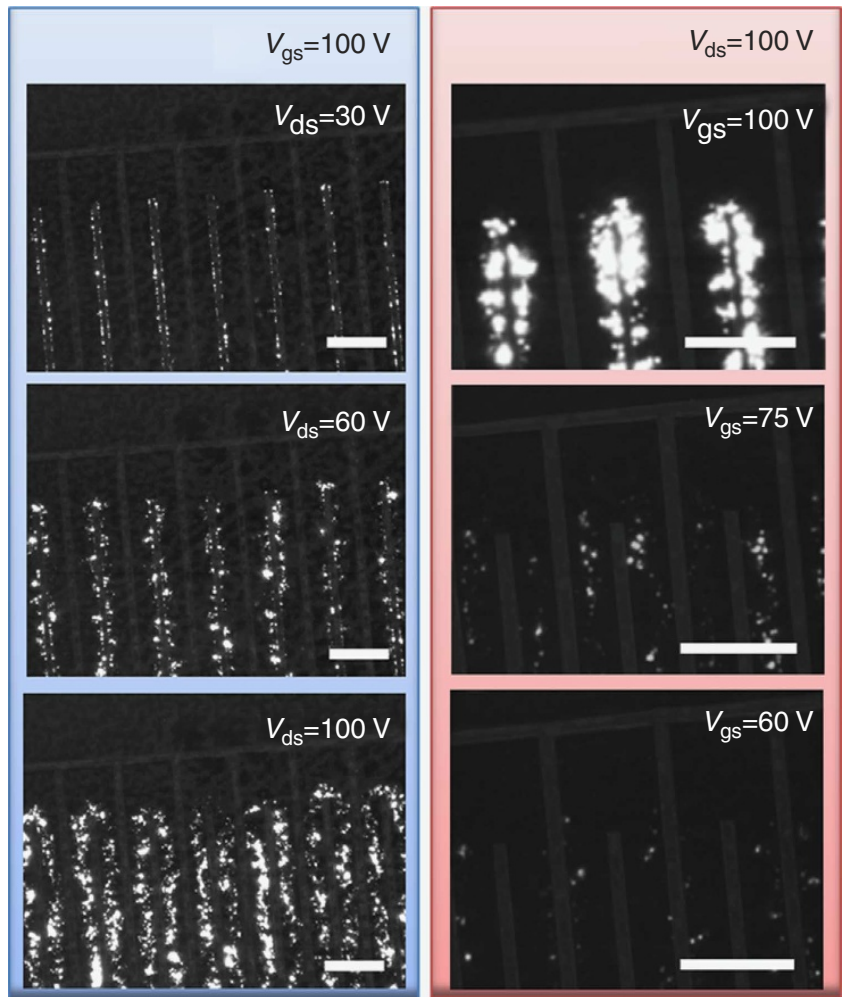

Figure 5 | Optical images of $\mathrm{CH}_{3} \mathrm{NH}_{3} \mathrm{Pbl}_{3}$ LE-FET emission zone at $\boldsymbol{T}=158 \mathrm{~K}$. (a-c) Frame images extracted from a video recorded while sweeping $V_{\text {ds }}$ from 0 to $100 \mathrm{~V}$ at constant $V_{\mathrm{gs}}=100 \mathrm{~V}$; the corresponding values of $V_{\mathrm{ds}}$ are indicated in the panels. (d-f) Frame images extracted from a video recorded while sweeping $V_{g s}$ from 0 to $100 \mathrm{~V}$ at constant $V_{\mathrm{ds}}=100 \mathrm{~V}$; the corresponding values of $V_{\mathrm{gs}}$ are indicated in the panels; note that the contrast of the metal contacts was slightly enhanced for clarity. See Supplementary Movies 1 and 2 for the source real-time videos of the measurements. Scale bars, $200 \mu \mathrm{m}$.

polycrystalline nature of the film (Fig. 1a,b), the EL emission zone can be clearly identified from the images. For a fixed gate bias of $V_{\mathrm{gs}}=100 \mathrm{~V}$ (Fig. $5 \mathrm{a}-\mathrm{c}$ ), the emission zone is mainly concentrated near the drain electrode when $V_{\mathrm{ds}}$ is small (Fig. 5a). This is due to the limited injection of holes resulting from the relative low absolute local gate potential at the drain electrode $\left|V_{\mathrm{d}}\right|$. By increasing $V_{\mathrm{ds}},\left|V_{\mathrm{d}}\right|$ increases, thus more holes are injected into the active channel and the EL intensity increases (Fig. 5b). Further increase of hole injection extends the emission area to the centre of the channel, enhancing the EL intensity even further (Fig. 5c). Conversely, for a fixed drain-source voltage of $V_{\mathrm{ds}}=100 \mathrm{~V}$ (Fig. 5d-f), the injected electron and hole current densities can no longer be regulated independently. Figure $5 \mathrm{~d}$ shows extremely bright emission from nearby the drain electrodes because of the overwhelming density of injected electrons recombining with a comparatively lower density of injected holes. Decreasing the gate voltage reduces the local gate potential at the source electrode $V_{\mathrm{s}}$ and increases $\left|V_{\mathrm{d}}\right|$, thus decreasing electron injection and increasing hole injection. This pushes the emission zone to the centre of the active channel and reduces the EL intensity as overall current density decreases (Fig. 5e). A further reduction of gate voltage pushes the emission zone closer to the source electrode, further weakening the EL intensity (see Fig. 5f). Continuous-frame videos showing the variation of EL intensity and position of the emission zone sweeping $V_{\mathrm{ds}}$ from 0 to $100 \mathrm{~V}$ at constant $V_{\mathrm{gs}}=100 \mathrm{~V}$ and sweeping $V_{\mathrm{gs}}$ from 0 to $100 \mathrm{~V}$ at constant $V_{\mathrm{ds}}=100 \mathrm{~V}$ are provided as Supplementary Movies 1 and 2. This demonstrates that full control of charge 
carrier injection and recombination in $\mathrm{CH}_{3} \mathrm{NH}_{3} \mathrm{PbI}_{3}$ LE-FET can be easily achieved by adjusting its biasing conditions.

In summary, we fabricated high-quality hybrid perovskite FETs and used them to determine intrinsic transport parameters of $\mathrm{CH}_{3} \mathrm{NH}_{3} \mathrm{PbI}_{3}$, which are of great relevance to electro-optic devices (including solar cells). Our main findings include the ambipolar nature of charge transport, the understanding of the origin and suppression of screening effects associated to the presence of ionic cations, the direct determination of electron and hole mobilities and their temperature dependence, and the effect of structural phase transition on the electronic properties of $\mathrm{CH}_{3} \mathrm{NH}_{3} \mathrm{PbI}_{3}$, all in good agreement with first-principle DFT calculations. Furthermore, bright EL owing to radiative recombination within the transistor channel was demonstrated under balanced charge injection. We believe this demonstration of a $\mathrm{CH}_{3} \mathrm{NH}_{3} \mathrm{PbI}_{3}$ LE-FET paves the way to the realization of solution-processed hybrid perovskite light-emitting devices such as high-brightness light-emitting diodes and electrical injection lasers. More work will be needed in this direction to minimize ionic screening, improve thin film crystallinity and optimize device architecture, for instance employing staggered FET configurations to increase carrier injection density ${ }^{47}$ or integrating surface microstructures for light management.

\section{Methods}

Perovskite deposition. The organic precursor methylammonium iodide $\mathrm{CH}_{3} \mathrm{NH}_{3} \mathrm{I}$ was synthetized by mixing $10 \mathrm{ml}$ of methylamine solution $\left(\mathrm{CH}_{3} \mathrm{NH}_{2}, 40 \%\right.$ in methanol, Tokyo Chemical Industry, Co., Ltd) and $14 \mathrm{ml}$ of hydroiodic acid (57\% wt in water, Sigma-Aldrich). The reaction was accomplished in ice bath for $2 \mathrm{~h}$ under magnetic stirring, and the solvent removed with a rotary evaporator $(1 \mathrm{~h}$ at $60 \mathrm{mbar}$ and $60^{\circ} \mathrm{C}$ ). The product was purified by dissolution in ethanol and recrystallization with diethylether, repeating the washing cycle six times. After filtration, the resulting white powder was dried in vacuum oven at $60^{\circ} \mathrm{C}$ for $24 \mathrm{~h}$. Thin film of $\mathrm{CH}_{3} \mathrm{NH}_{3} \mathrm{PbI}_{3}$ deposited on clean electrodes pre-patterned $\mathrm{SiO}_{2}$ substrates. A $20 \%$ wt $\mathrm{CH}_{3} \mathrm{NH}_{3} \mathrm{PbI}_{3}$ solution was prepared mixing stoichiometric amounts of $\mathrm{CH}_{3} \mathrm{NH}_{3} \mathrm{I}$ and $\mathrm{PbI}_{2}(99 \%$, Sigma-Aldrich) in a solvent mixture of $\gamma$-butyrolactone and dimethylsulfoxide (7:3 volume ratio) and stirred overnight at $100^{\circ} \mathrm{C}$. To obtain continuous and uniform films, the solvent engineering technique was used ${ }^{14}$. The solution was spin-coated on the substrate using a two-step ramp: 1,000 r.p.m. for 10 s, 5,000 r.p.m. for 20 s.

Toluene was drop-casted on the substrate during the second step. The resulting film was finally annealed at $100^{\circ} \mathrm{C}$ for $30 \mathrm{~min}$.

Perovskite characterization. Morphological analysis was performed with a FEI Helios 650 Nanolab SEM with $10 \mathrm{KV}$ acceleration voltage and a scanning probe microscope Digital Instrument Dimension V (AFM analysis). The software WSxM was used for editing and plotting of the AFM images. The X-ray diffraction structural spectra were obtained using a diffractometer BRUKER D8 ADVANCE with Bragg-Brentano geometry employing $\mathrm{Cu} \mathrm{K} \alpha$ radiation $(l=1.54,056 \AA)$, a step increment of $0.02^{\circ}, 1 \mathrm{~s}$ of acquisition time and sample rotation of $5 \mathrm{~min}^{-1}$.

FET fabrication. Heavily p-doped $\mathrm{Si}$ substrates with thermally grown $\mathrm{SiO}_{2}$ $(500 \mathrm{~nm})$ layer were cleaned by two rounds of sonication in acetone and iso-propyl alcohol $(20 \mathrm{~min}$ each round, and then dried under nitrogen flow. Interdigitated electrodes $(L=80$ and $100 \mu \mathrm{m}, W=20 \mathrm{~mm})$ were patterned using conventional photolithography. Electrodes of $\mathrm{Ni}(10 \mathrm{~nm})$ and $\mathrm{Au}(50 \mathrm{~nm})$ were thermally evaporated. The substrates were then undergoing lift-off process to obtain the desired electrodes. Before the spin coating of the active materials, an oxygen plasma cleaning treatment was performed on the substrate, for $1 \mathrm{~min}$, to improve the wetting of the surface and obtain flatter and homogeneous perovskite thin film (see perovskite deposition).

Temperature-dependent FET measurements. FET devices were mounted into a liquid nitrogen-cooled Linkam Stage (FTIR 600) that allow to scan FET operating temperature of the device from $300 \mathrm{~K}$ down to $77 \mathrm{~K}$. The FET electrical characteristics were acquired with Agilent B2902A Precision Source/Measure Unit in dark environment. The data were then analysed with OriginPro software.

EL measurement. The EL spectra were acquired using the Nikon eclipse LV100 microscope with LU plan fluor $\times 10$ objectives, whereas the FET were enclosed in the Linkam Stage and FET electrical behaviour was controlled using Agilent B2902A Precision Source/Measure Unit. EL emission signal was focused into optic fibre that coupled to USB2000 Ocean Optics to record EL spectra. All EL spectra were measured with $1 \mathrm{~s}$ integration time over three averages. The optical images and videos were taken and acquired by Thorlabs DCC1545M High-Resolution USB2.0 CMOS Camera with weak illumination to enhance the optical contrast

Computational method. The DFT calculations have been carried out by the Perdew-Burke-Ernzerhof generalized gradient approximation using plane-wave self-consistent field (PWSCF) code implemented in the Quantum ESPRESSO package ${ }^{62}$. For the structural optimization and band structure calculations, ultrasoft pseudopotentials including scalar-relativistic or full-relativistic effect were used to describe electron-ion interactions with electronic orbitals of $\mathrm{H}\left(1 \mathrm{~s}^{1}\right) ; \mathrm{O}, \mathrm{N}$ and $\mathrm{C}\left(2 \mathrm{~s}^{2}, 2 \mathrm{p}^{2}\right) ; \mathrm{I}\left(5 \mathrm{~s}^{2}, 5 \mathrm{p}^{2}\right)$ and $\mathrm{Pb}\left(5 \mathrm{~d}^{10}, 6 \mathrm{~s}^{2}, 6 \mathrm{p}^{2}\right)^{63}$. The plane wave energy cutoff of wave function (charge) was set to be 40 (300) Ry. The crystal cell parameters were $a=b=8.81 \AA$, and $c=12.99 \AA$ for tetragonal phase (I $4 / \mathrm{mcm}$ space group); and $a=8.77 \AA, b=8.56 \AA$ and $c=12.97 \AA$ for the orthorhombic phase (Pnma space group) of bulk $\mathrm{CH}_{3} \mathrm{NH}_{3} \mathrm{PbI}_{3}$. The Monkhort-Pack scheme $\mathrm{k}$-meshes are $4 \times 4 \times 4$ for these two phases. The crystal cell and atomic positions were optimized until forces on single atoms were smaller than $0.01 \mathrm{eV} \AA^{-1}$. The molecular graphics viewer VESTA was used to plot molecular structures.

The effective masses for electron $\left(m_{e}^{*}\right)$ and hole $\left(m_{h}^{*}\right)$ were estimated by fitting of the dispersion relation of $m^{*}=\hbar^{2}\left[\frac{\partial^{2} \varepsilon(k)}{\partial k^{2}}\right]^{-1}$ from band structures in Fig. 3 along the directions $\Gamma-\mathrm{X}, \Gamma-\mathrm{Z}$ and $\Gamma-\mathrm{M}$ for tetragonal phase and $\Gamma-\mathrm{X}$ and $\Gamma-\mathrm{Z}$ for orthorhombic phase together with average values in these different routes. The carrier lifetime was evaluated by the semi-classical Boltzmann transport theory ${ }^{49}$. The only contribution of acoustic phonons was considered in evaluating scattering lifetime, where the charge carrier density $(n)$ and mobility $(\mu)$ are approximated as ${ }^{27,64}$

$$
\begin{gathered}
n=\frac{\left(2 m^{*} k_{\mathrm{B}} T\right)^{3 / 2}}{3 \pi^{2} \hbar^{3}}{ }^{0} F_{0}^{3 / 2} \\
\mu=\frac{2 \pi \hbar^{4} e B}{m_{I}^{*}\left(2 m_{\mathrm{b}}^{*} k_{\mathrm{B}} T\right)^{3 / 2} \Xi^{2}} \frac{3^{0} F_{-2}^{1}}{{ }^{0} F_{0}^{3 / 2}} \\
\text { where }{ }^{n} F_{l}^{m}=\int_{0}^{\infty}\left(-\frac{\partial f}{\partial \zeta}\right) \zeta^{n}\left(\zeta+\alpha \zeta^{2}\right)^{m}\left[(1+2 \alpha \zeta)^{2}+2\right]^{l / 2} d \zeta \\
f=1 /\left(e^{\zeta-\xi}+1\right) \\
\alpha=k_{\mathrm{B}} T / E_{\mathrm{g}}
\end{gathered}
$$

$k_{\mathrm{B}}$ is the Boltzmann constant, $e$ is the elementary charge, $T$ is the temperature, $\hbar$ is the Planck constant and $\xi$ is the reduced chemical potential; $m^{*}$ is the density of state effective mass, $m_{\mathrm{I}}^{*}$ is the conductivity effective mass, $m_{\mathrm{b}}^{*}$ is the band effective mass; $B$ is the bulk modulus $\left(B=\partial^{2} E / \partial V^{2}\right), \Xi_{e-p / h-p}$ is the electron - phonon (or hole - phonon) coupling energy $\left(\Xi_{e-p / h-p}=V_{0}\left(\Delta E_{C B M / V B M} / \Delta V\right), n, m\right.$ and $l$ power integer indices, $E_{\mathrm{g}}$ is the electronic band gap and $\zeta$ the reduced carrier energy. Note that the generalized gradient approximation method including spinorbital coupling yields largely underestimated values of the band gaps. Detailed estimate of the band gap values would require many-body perturbation theory (GW method) ${ }^{49,50}$. However, as the band structure is not significantly affected by GW correction, our calculations of the effective mass and mobility are still reliable.

\section{References}

1. Green, M. A., Ho-Baillie, A. \& Snaith, H. J. The emergence of perovskite solar cells. Nat. Photon 8, 506-514 (2014).

2. Gao, P., Gratzel, M. \& Nazeeruddin, M. K. Organohalide lead perovskites for photovoltaic applications. Energy Environ. Sci. 7, 2448-2463 (2014).

3. Lee, M., Miyasaka, T., Murakami, T., Snaith, H. \& Teuscher, J. Efficient hybrid solar cells based on meso-superstructured organometal halide perovskites. Science 338, 643-647 (2012).

4. Stranks, S. D. et al. Electron-hole diffusion lengths exceeding 1 micrometer in an organometal trihalide perovskite absorber. Science 342, 341-344 (2013).

5. Xing, G. et al. Long-range balanced electron- and hole-transport lengths in organic-inorganic $\mathrm{CH}_{3} \mathrm{NH}_{3} \mathrm{PbI}_{3}$. Science 342, 344-347 (2013).

6. D'Innocenzo, V., Srimath Kandada, A. R., De Bastiani, M., Gandini, M. \& Petrozza, A. Tuning the light emission properties by band gap engineering in hybrid lead halide perovskite. J. Am. Chem. Soc. 136, 17730-17733 (2014).

7. Gonzalez-Pedro, V. et al. General working principles of $\mathrm{CH}_{3} \mathrm{NH}_{3} \mathrm{PbX}_{3}$ perovskite solar cells. Nano Lett. 14, 888-893 (2014).

8. Liu, M., Johnston, M. B. \& Snaith, H. J. Efficient planar heterojunction perovskite solar cells by vapour deposition. Nature 501, 395-398 (2013).

9. Zhou, H. et al. Interface engineering of highly efficient perovskite solar cells. Science 345, 542-546 (2014).

10. Kim, H.-S., Im, S. H. \& Park, N.-G. Organolead halide perovskite: new horizons in solar cell research. J. Phys. Chem. C 118, 5615-5625 (2014).

11. Kojima, A., Teshima, K., Shirai, Y. \& Miyasaka, T. Organometal halide perovskites as visible-light sensitizers for photovoltaic cells. J. Am. Chem. Soc. 131, 6050-6051 (2009). 
12. Burschka, J. et al. Sequential deposition as a route to high-performance perovskite-sensitized solar cells. Nature 499, 316-319 (2013).

13. Boix, P. P., Nonomura, K., Mathews, N. \& Mhaisalkar, S. G. Current progress and future perspectives for organic/inorganic perovskite solar cells. Mater. Today 17, 16-23 (2014).

14. Jeon, N. J. et al. Solvent engineering for high-performance inorganic-organic hybrid perovskite solar cells. Nat. Mater. 13, 897-903 (2014).

15. Heo, J. H. et al. Efficient inorganic-organic hybrid heterojunction solar cells containing perovskite compound and polymeric hole conductors. Nat. Photon 7, 486-491 (2013)

16. Salim, T. et al. Perovskite-based solar cells: impact of morphology and device architecture on device performance. J. Mater. Chem. A 3, 8943-8969 (2015).

17. Luo, J. et al. Water photolysis at $12.3 \%$ efficiency via perovskite photovoltaics and Earth-abundant catalysts. Science 345, 1593-1596 (2014).

18. Tan, Z.-K. et al. Bright light-emitting diodes based on organometal halide perovskite. Nat. Nano 9, 687-692 (2014).

19. Gil-Escrig, L. et al. Efficient photovoltaic and electroluminescent perovskite devices. Chem. Commun. 51, 569-571 (2015).

20. Hoye, R. L. Z. et al. Enhanced performance in fluorene-free organometal halide perovskite light-emitting diodes using tunable, low electron affinity oxide electron injectors. Advan. Mater. 27, 1414-1419 (2015).

21. Sutherland, B. R., Hoogland, S., Adachi, M. M., Wong, C. T. O. \& Sargent, E. H. Conformal organohalide perovskites enable lasing on spherical resonators. ACS Nano 8, 10947-10952 (2014).

22. Deschler, F. et al. High photoluminescence efficiency and optically pumped lasing in solution-processed mixed halide perovskite semiconductors. J. Phys. Chem. Lett. 5, 1421-1426 (2014).

23. Xing, G. et al. Low-temperature solution-processed wavelength-tunable perovskites for lasing. Nat. Mater. 13, 476-480 (2014).

24. Edri, E. et al. Elucidating the charge carrier separation and working mechanism of CH3NH3PbI3 - xClx perovskite solar cells. Nat. Commun. 5, 3461 (2014).

25. Bergmann, V. W. et al. Real-space observation of unbalanced charge distribution inside a perovskite-sensitized solar cell. Nat. Commun. 5, 5001 (2014).

26. Giorgi, G., Fujisawa, J.-I., Segawa, H. \& Yamashita, K. Small photocarrier effective masses featuring ambipolar transport in methylammonium lead iodide perovskite: a density functional analysis. J. Phys. Chem. Lett. 4, 4213-4216 (2013).

27. He, Y. P. \& Galli, G. Perovskites for solar thermoelectric applications: a first principle study of $\mathrm{CH}_{3} \mathrm{NH}_{3} \mathrm{Al}_{3}(\mathrm{~A}=\mathrm{Pb}$ and $\mathrm{Sn})$. Chem. Mater. 26, 5394-5400 (2014).

28. Wehrenfennig, C., Eperon, G. E., Johnston, M. B., Snaith, H. J. \& Herz, L. M. High charge carrier mobilities and lifetimes in organolead trihalide perovskites. Advan. Mater. 26, 1584-1589 (2014).

29. Stoumpos, C. C., Malliakas, C. D. \& Kanatzidis, M. G. Semiconducting tin and lead iodide perovskites with organic cations: phase transitions, high mobilities, and near-infrared photoluminescent properties. Inorg Chem. 52, 9019-9038 (2013).

30. Dong, Q. et al. Electron-hole diffusion lengths $>175 \mu \mathrm{m}$ in solution grown $\mathrm{CH}_{3} \mathrm{NH}_{3} \mathrm{PbI}_{3}$ single crystals. Science 347, 967-970 (2015).

31. Shi, D. et al. Low trap-state density and long carrier diffusion in organolead trihalide perovskite single crystals. Science 347, 519-522 (2015).

32. Nie, W. et al. High-efficiency solution-processed perovskite solar cells with millimeter-scale grains. Science 347, 522-525 (2015).

33. Xiao, Z. et al. Giant switchable photovoltaic effect in organometal trihalide perovskite devices. Nat. Mater. 14, 193-198 Advance online publication (2014).

34. Frost, J. M., Butler, K. T. \& Walsh, A. Molecular ferroelectric contributions to anomalous hysteresis in hybrid perovskite solar cells. APL Mater. 2, 081506 (2014).

35. Snaith, H. J. et al. Anomalous hysteresis in perovskite solar cells. J. Phys. Chem. Lett. 5, 1511-1515 (2014).

36. Shao, Y., Xiao, Z., Bi, C., Yuan, Y. \& Huang, J. Origin and elimination of photocurrent hysteresis by fullerene passivation in $\mathrm{CH}_{3} \mathrm{NH}_{3} \mathrm{PbI}_{3}$ planar heterojunction solar cells. Nat. Commun. 5, 5784 (2014).

37. Unger, E. L. et al. Hysteresis and transient behavior in current-voltage measurements of hybrid-perovskite absorber solar cells. Energy Environ. Sci. 7, 3690-3698 (2014).

38. Kagan, C. R., Mitzi, D. B. \& Dimitrakopoulos, C. D. Organic-inorganic hybrid materials as semiconducting channels in thin-film field-effect transistors. Science 286, 945-947 (1999).

39. Mitzi, D. B., Dimitrakopoulos, C. D. \& Kosbar, L. L. Structurally tailored organic - inorganic perovskites: optical properties and solution-processed channel materials for thin-film transistors. Chem. Mater. 13, 3728-3740 (2001).

40. Mitzi, D. B. et al. Hybrid field-effect transistor based on a low-temperature melt-processed channel layer. Advan. Mater. 14, 1772-1776 (2002).

41. Noh, J. H., Im, S. H., Heo, J. H., Mandal, T. N. \& Seok, S. I. Chemical management for colorful, efficient, and stable inorganic-organic hybrid nanostructured solar cells. Nano Lett. 13, 1764-1769 (2013).
42. Capelli, R. et al. Organic light-emitting transistors with an efficiency that outperforms the equivalent light-emitting diodes. Nat. Mater. 9, 496-503 (2010).

43. Verlaak, S., Cheyns, D., Debucquoy, M., Arkhipov, V. \& Heremans, P. Numerical simulation of tetracene light-emitting transistors: a detailed balance of exciton processes. Appl. Phys. Lett. 85, 2405 (2004).

44. Egger, D. A., Edri, E., Cahen, D. \& Hodes, G. Perovskite solar cells: Do we know what we do not know? J. Phys. Chem. Lett. 6, 279-282 (2015).

45. Kutes, Y. et al. Direct observation of ferroelectric domains in solution-processed $\mathrm{CH}_{3} \mathrm{NH} 3 \mathrm{PbI}_{3}$ perovskite thin films. J. Phys. Chem. Lett. 5, 3335-3339 (2014)

46. Wang, Q. et al. Qualifying composition dependent $\mathrm{p}$ and $\mathrm{n}$ self-doping in $\mathrm{CH}_{3} \mathrm{NH}_{3} \mathrm{PbI}_{3}$. Appl. Phys. Lett. 105, 163508 (2014).

47. Zaumseil, J. \& Sirringhaus, H. Electron and ambipolar transport in organic field-effect transistors. Chem. Rev. 107, 1296-1323 (2007).

48. Amat, A. et al. Cation-induced band-gap tuning in organohalide perovskites: interplay of spin-orbit coupling and octahedra tilting. Nano Lett. 14, 3608-3616 (2014).

49. Wang, H., Pei, Y. Z., LaLonde, A. D. \& Snyder, G. J. Weak electron-phonon coupling contributing to high thermoelectric performance in n-type PbSe. Proc Natl Acad. Sci. USA 109, 9705-9709 (2012).

50. Umari, P., Mosconi, E. \& De Angelis, F. Relativistic GW calculations on $\mathrm{CH}_{3} \mathrm{NH}_{3} \mathrm{PbI}_{3}$ and $\mathrm{CH}_{3} \mathrm{NH}_{3} \mathrm{SnI}_{3}$ perovskites for solar cell applications. Sci. Rep. 4, 4467 (2014)

51. Ma, J. \& Wang, L. W. Nanoscale charge localization induced by random orientations of organic molecules in hybrid perovskite $\mathrm{CH}_{3} \mathrm{NH}_{3} \mathrm{PbI}_{3}$. Nano Lett. 15, 248-253 (2014).

52. Frost, J. M. et al. Atomistic origins of high-performance in hybrid halide perovskite solar cells. Nano Lett. 14, 2584-2590 (2014).

53. Juarez-Perez, E. J. et al. Photoinduced giant dielectric constant in lead halide perovskite solar cells. J. Phys. Chem. Lett. 5, 2390-2394 (2014).

54. Bisri, S. Z., Piliego, C., Gao, J. \& Loi, M. A. Outlook and Emerging Semiconducting Materials for Ambipolar Transistors. Advanced Materials 26, 1176-1199 (2014).

55. Swensen, J. S., Soci, C. \& Heeger, A. J. Light emission from an ambipolar semiconducting polymer field-effect transistor. Appl. Phys. Lett. 87, 253511 (2005)

56. Muccini, M. A bright future for organic field-effect transistors. Nat. Mater. 5, 605-613 (2006).

57. Chen, Z. Y. et al. Origin of the different transport properties of electron and hole polarons in an ambipolar polyselenophene-based conjugated polymer. Phys. Rev. B 84, 115211 (2011).

58. Wu, K. W. et al. Temperature-dependent excitonic photoluminescence of hybrid organometal halide perovskite films. Phys. Chem. Chem. Phys. 16, 22476-22481 (2014).

59. Fang, H.-H. et al. Photophysics of organic-inorganic hybrid lead iodide perovskite single crystals. Advan. Funct. Mater., Published online (2015).

60. Even, J., Pedesseau, L. \& Katan, C. Analysis of multivalley and multibandgap absorption and enhancement of free carriers related to exciton screening in hybrid perovskites. J. Phys. Chem. C 118, 11566-11572 (2014).

61. Wang, Y. et al. Density functional theory analysis of structural and electronic properties of orthorhombic perovskite $\mathrm{CH}_{3} \mathrm{NH}_{3} \mathrm{PbI}_{3}$. Phys. Chem. Chem. Phys. 16, 1424-1429 (2014).

62. Giannozzi, P. et al. QUANTUM ESPRESSO: a modular and open-source software project for quantum simulations of materials. J. Phys. Condens. Mat. 21, 395502 (2009).

63. Garrity, K. F., Bennett, J. W., Rabe, K. M. \& Vanderbilt, D. Pseudopotentials for high-throughput DFT calculations. Comp. Mater. Sci. 81, 446-452 (2014).

64. Ravich, Y. I., Efimova, B. A. \& Tamarche, V. i. Scattering of Current Carriers and Transport Phenomena in Lead Chacogenides. Phys. Status Solidi B 43, 11-33 (1971).

\section{Acknowledgements}

Research was supported by NTU (NAP startup grant M4080511), the Singapore Ministry of Education (MOE2013-T2-044 and MOE2011-T3-1-005) and the Singapore-Berkeley Research Initiative for Sustainable Energy (SinBeRISE) CREATE Programme. We are grateful to Nripan Mathews, Pablo Boix, Mario Caironi and Annamaria Petrozza for the useful discussions, to Stefano Vezzoli and Saleem Umar for their help with electroluminescence measurements, and to Liu Hailong for assistance with SEM imaging.

\section{Author contributions}

C.S. and X.Y.C. generated the original idea and designed the experiment. X.Y.C. fabricated the FET devices and gathered all transport and electroluminescence data. D.C. synthesized the perovskite precursors, prepared and characterized the films. J.Y. performed DFT modelling and mobility calculations. A.B. analysed the electroluminescence data. All the authors contributed to interpretation of the results and writing of the manuscript. A.B. and C.S. supervised the work.

\section{Additional information}

Supplementary Information accompanies this paper at http://www.nature.com/ naturecommunications 
Competing financial interests: The authors declare no competing financial interests.

Reprints and permission information is available online at http://npg.nature.com/ reprintsandpermissions/

How to cite this article: Chin, X.Y. et al. Lead iodide perovskite light-emitting field-effect transistor. Nat. Commun. 6:7383 doi: 10.1038/ncomms8383 (2015). (c) (i) This work is licensed under a Creative Commons Attribution 4.0 International License. The images or other third party material in this article are included in the article's Creative Commons license, unless indicated otherwise in the credit line; if the material is not included under the Creative Commons license, users will need to obtain permission from the license holder to reproduce the material. To view a copy of this license, visit http://creativecommons.org/licenses/by/4.0/ 